\title{
COMPUTATIONAL FLUID DYNAMICS SIMULATIONS OF FLOW IN THE RENAL ARTERIES AFTER STENT GRAFT IMPLANTATION
}

\author{
Tianyi Xia, Matthew G. Doyle \\ Dept. of Mechanical and Industrial \\ Engineering, University of Toronto \\ Toronto, Canada
}

\author{
Thomas L. Forbes \\ Division of Vascular Surgery, \\ UHN, and University of Toronto \\ Toronto, Canada
}

\author{
Cristina H. Amon \\ Dept. of Mech. and Industrial Eng., \\ and IBBME, University of Toronto \\ Toronto, Canada
}

\begin{abstract}
The objective of this work is to report a computational fluid dynamics study assessing the hemodynamic effects of fenestration misalignment, towards understanding post-surgical complications of fenestrated endovascular aneurysm repair for abdominal aortic aneurysms. Idealized models were constructed based on geometries from a patient with an infrarenal aortic aneurysm. Fenestrated stent grafts were simulated in the models, with combinations of different fenestration misalignments and takeoff angles. Computational fluid dynamics simulations were performed by solving the governing equations for blood flow under physiologically realistic boundary conditions. Hemodynamic results of renal artery flow rate and time-averaged wall shear stresses were analyzed to build connections between the degree of fenestration misalignment, the takeoff angle, and changes in flow dynamics.
\end{abstract}

Keywords-abdominal aortic aneurysms; computational fluid dynamics; fenestrated endovascular aneurysm repair; hemodynamics; time-averaged wall shear stress

\section{INTRODUCTION}

An abdominal aortic aneurysm (AAA) is a weakening and progressive enlargement of the aorta, which is the body's main artery. If left untreated, AAAs will continue to grow and potentially rupture, leading to death in $80 \%$ to $90 \%$ of cases [1]. Fenestrated endovascular aneurysm repair (FEVAR) is a minimally invasive treatment option for AAAs that involve the branch vessels such as the renal arteries. FEVAR involves inserting a fenestrated stent graft into the aorta at the site of the aneurysm and can include additional stenting of the branch vessels. FEVAR has been continuously evolving over the past two decades [2]; however, post-surgical complications including acute kidney injury (AKI) and renal artery thrombosis [3] were reported, and in some cases, at greater rates than for open aneurysm repair [3], leading to secondary interventions and renal function deterioration [4]. Some cases of post-surgical renal dysfunction were associated with aortic stent graft misalignment [4], where the fenestration was not aligned with the renal artery ostium; however, associations between fenestration misalignment and post-surgical complications have not been investigated in vivo due to difficulties with data acquisition [5].
As a first step towards understanding post-surgical complications of FEVAR, including AKI and renal artery thrombosis, this study aims to use computational fluid dynamics (CFD) to determine how fenestration misalignment impacts renal artery hemodynamics. Two factors, renal artery flow rate and renal artery time-averaged wall shear stress (TAWSS) were adopted as hemodynamics indices [6-10] to indicate the risks of AKI and renal thrombosis.

\section{METHODS}

\section{A. Geometry}

An idealized 3-D computational model of a post-surgical AAA was built using ANSYS DesignModeler (ANSYS Inc., Canonsburg, PA, USA). The lengths and diameters of the vessels were taken from the geometry of a patient with an infrarenal aortic aneurysm [11]. The patient was treated with endovascular aneurysm repair instead of FEVAR. However, this prototype was chosen due to availability of both the AAA geometry and physiological boundary conditions. It is assumed that neither the post-surgical model boundary conditions nor the post-surgical geometry changes with aortic aneurysm type. Taking advantage of the assumed symmetry between the left and right sides of the model, a half-symmetric geometry was adopted, including half of the aorta, one renal artery, and one iliac artery (Fig. 1). A fenestrated stent graft was then simulated in the model, with a flared renal stent that opens in the renal artery.

Both a reduction of the effective area of the renal artery orifice and the takeoff angle (TOA) between the aorta and the renal artery were considered in our model. Reduction of effective area on the proximal end of renal artery is caused by fenestration misalignment [4]. TOA is defined as the angle between the centreline of the renal stent and centreline axis of aorta [4].

A total of 15 cases were simulated, consisting of all combinations of five different fenestration alignments and three different TOAs. A fully aligned case, along with four cases of misalignment were considered. For the misaligned cases, the fenestration was positioned either $2.8 \mathrm{~mm}$ above or $2.8 \mathrm{~mm}$ below the proximal end of the renal artery, referred to as 'vertical misalignment', or rotated relative to the proximal end of the renal artery by either $17.5^{\circ}$ clockwise or $17.5^{\circ}$ counterclockwise, referred to as 'horizontal misalignment' 

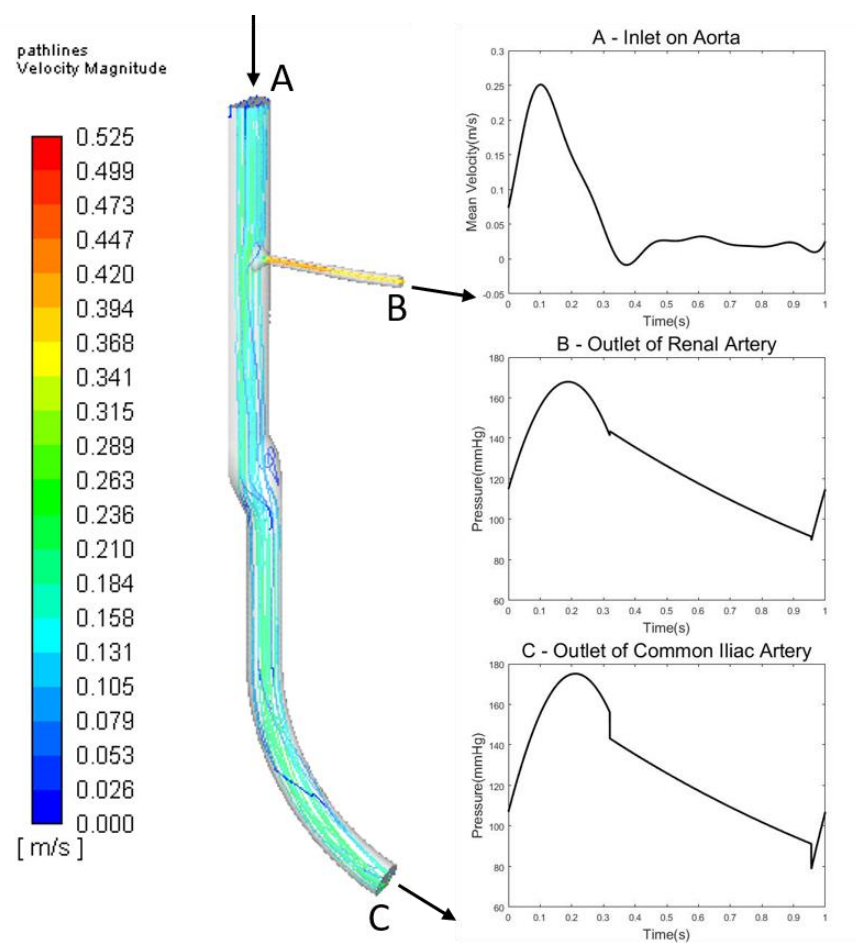

Figure 1. Post-surgical model with boundary conditions and velocity pathlines

(Fig. 2). These four misalignment cases were designed to have the equivalent of $45 \%$ effective area reduction in the proximal end of renal artery.

For the aligned case and each misaligned case, three different TOA were considered: $87.25^{\circ}$ to model the average TOA, and $60.95^{\circ}$ and $113.55^{\circ}$ to model the maximum and minimum TOAs (Fig. 3), as reported by Jiale et al. [4].

\section{B. Boundary Conditions}

Patient-specific flow data were considered to define the inlet and outlet boundary conditions. Supraceliac-level flow rates, acquired from cine phase-contrast magnetic resonance imaging (PC-MRI) [11], were adapted to a Womersley velocity profile [12] applied at the inlet.

For the outlets of the renal and iliac arteries, 2-element Windkessel models [13] were used. These models account for arterial compliance and total peripheral resistance through analogies between fluid flow and electric circuits. The parameters for these models were fit to pressure data for the renal and iliac artery, approximated from brachial pressure cuff measurements immediately after the PC-MRI scan (Fig. 4) [11].

No-slip boundary conditions were specified for all vessel and stent graft walls, which were assumed to be rigid. A symmetry boundary condition was applied to the centre of the aorta. To delineate the effects of fenestration misalignment on renal artery flow, identical boundary conditions were defined for all simulations. Blood was assumed to be non-Newtonian fluid based on Quesmada viscosity model.
A

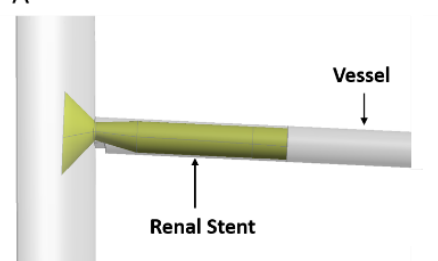

B

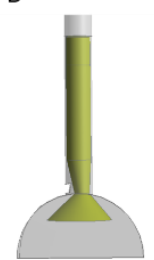

Figure 2. Post-surgical geometry with (A) vertical misalignment; and (B) horizontal misalignment.

A

A
C
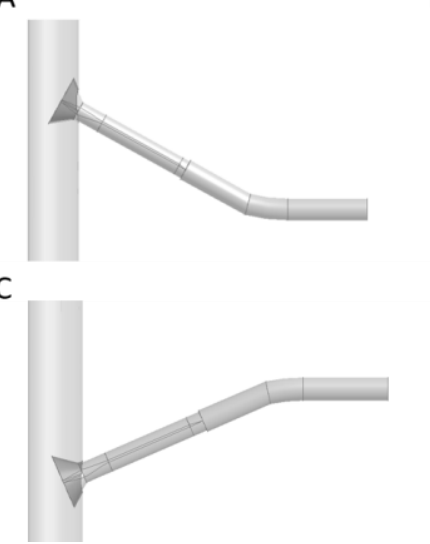

Figure 3. (A) Minimum (60.95 $)$, (B) mean $\left(87.25^{\circ}\right)$, and (C) maximum $\left(113.55^{\circ}\right)$ takeoff angles

\section{Numerical Analysis}

Simulation of pulsatile flow in this 3-D post-surgical geometry were performed by solving the continuity and momentum equations using a finite volume approach in ANSYS FLUENT v. 18.

The computational geometries were discretized by tetrahedral patch-conforming methods with advanced functions of proximity and curvature in ANSYS ICEM CFD. Mesh layers were refined on the walls of the renal arteries to accurately resolve the renal artery WSS.

Mesh independence studies were carried out for two steady state simulations, conducted at $t=0.1 \mathrm{~s}$, corresponding to the maximum inlet velocity, and $t=0.5 \mathrm{~s}$, corresponding to a low inlet velocity and high outlet pressure. With the number of elements increasing by 50\% for each refinement, 6 different mesh sizes were applied on each geometry, and grid independence was deemed to be reached when both the change of renal artery flow rate and the change of renal artery WSS were within $\pm 5 \%$ between two successive meshes. Among the 15 studied geometries, element numbers required to achieve grid independence varied from 199,708 to 450,033 .

Relative residuals of continuity and velocities in the $x, y$, and $z$ coordinates were set to $1 \times 10^{-4}$. Based on monitoring the Courant number in pulsatile flow simulations, a uniform $0.02 \mathrm{~s}$ time-step was adopted. As suggested by Kandail et al. [7], simulations for all geometries were performed for four cardiac cycles to achieve periodic results. 


\section{RESULTS AND DISCUSSIONS}

Renal artery flow rate and renal artery TAWSS were used in our study as indices of risks of AKI and renal thrombosis, respectively. A decrease of renal artery flow rate leads to a decrease in kidney blood supply; hence it is an indicator of potential AKI. A decrease in renal TAWSS is suggestive of increased chance of renal thrombosis according to the Low Shear Theory [6].

\section{A. Comparison of Renal Artery Flow Rate between Misaligned and Aligned Cases}

Renal artery flow rates between cases with aligned and misaligned fenestrations were compared to determine the influence of fenestration misalignment on the amount of blood flow to the kidney. As is summarized in Table 1 and Fig. 4, a decrease of renal artery flow rate was observed by comparing flow rates in the misaligned models and the aligned model with the same TOA. The maximum decrease was found in the case where the fenestration was $2.8 \mathrm{~mm}$ below the proximal renal artery with a TOA of $60.95^{\circ}(11.0 \%)$, while the minimum was found where the fenestration was $2.8 \mathrm{~mm}$ above the renal artery with a TOA of $113.55^{\circ}(7.1 \%)$. Fenestration misalignment caused a decrease of renal artery flow rate in all studied renal TOAs, hence an increased opportunity for post-surgical renal AKI.

\section{B. Comparison of Renal Flow Rate between Different TOAs}

Results of renal flow rates for different TOAs were studied to determine the influence of renal artery position on renal flow rate. As shown in Fig. 4, for each group with the same alignment, the case with the $60.95^{\circ} \mathrm{TOA}$ had a higher renal flow rate than the case with the $87.25^{\circ} \mathrm{TOA}$; while the case with the $113.55^{\circ}$ TOA had the lowest renal artery flow rate. Within misaligned cases, by comparing renal flow rates of cases with $133.55^{\circ}$ TOAs to cases with $60.95^{\circ}$ TOAs, the maximum decrease of flow rate was found in the case where the fenestration was rotated $17.5^{\circ}$ clockwise $(18.0 \%)$, while the minimum was found where the fenestration was $2.8 \mathrm{~mm}$ below the renal artery (17.4\%). Comparing to the percentage changes due to misalignment effects on renal flow rate (maximum of $11.0 \%$ ), change of TOA had a larger effect on the decrease of renal flow rate.

\section{Comparison of Renal TAWSS between Misaligned and Aligned Cases}

Renal artery TAWSS between cases with aligned and misaligned fenestrations were compared to delineate the influence of fenestration misalignment on TAWSS. As is summarized in Table 2 and Fig. 5, decrease of renal artery TAWSS was observed by comparing cases of the misalignment models against the aligned models with constant TOAs. The maximum percentage decrease was found in the case where the fenestration was $2.8 \mathrm{~mm}$ below the renal artery with a TOA of $87.25^{\circ}(21.0 \%)$, while the minimum was found where the fenestration was rotated $17.5^{\circ}$ counterclockwise with a TOA of $60.95^{\circ}(17.7 \%)$.

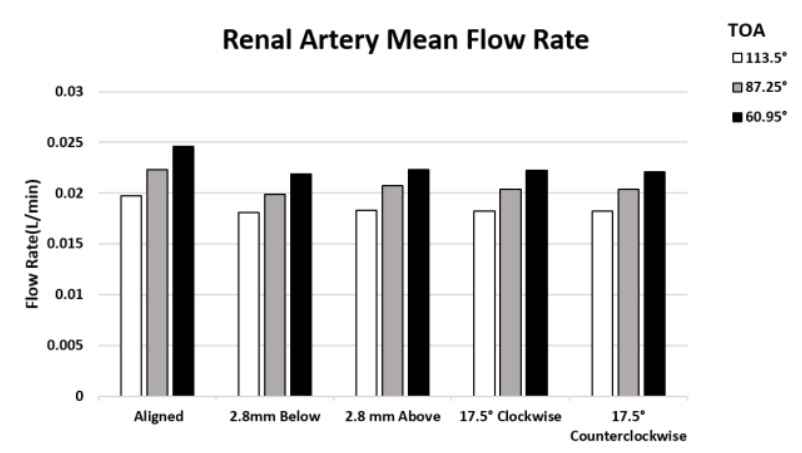

Figure 4. Renal artery flow rate $(\mathrm{L} / \mathrm{min})$ in different misalignment cases.

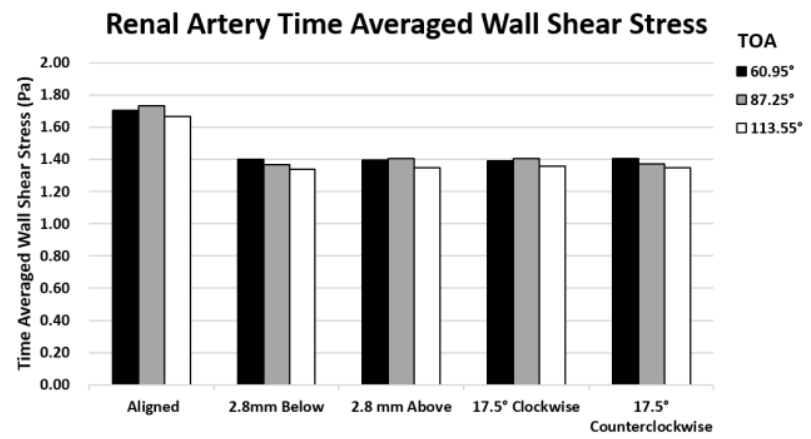

Figure 5 Renal artery time averaged wall shear stress in different misalignment cases.

In our study, we showed that fenestration misalignment and changes in TOA cause changes in renal hemodynamics, including the decrease of renal flow rate and renal TAWSS, which are suggestive of increased risk for post-surgical complications, including AKI and renal thrombosis.

Georgakarakos et al. [14] studied the impact of fenestration misalignment on renal WSS, but without considering the flaring of the renal stents or the reduction of the effective area of the proximal renal stent. In our institution, flaring of the renal stent is standard practice during FEVAR. Kandail et al. [7, 15] simulated blood flow with different TOAs and studied effects of flaring stent graft, but without considering either reduction of effective area or its combined effect with changes in TOA.

We were surprised to find that misalignment cases with constant reduction of effective renal artery area had different reductions in renal artery flow compared to the aligned case. Within the 2 cases of vertical misalignment (the fenestration above or below the renal artery) and the 2 cases of horizontal misalignment (the fenestration rotated either clockwise or counterclockwise), the case with the fenestration below the renal artery caused the largest decrease in renal artery flow, while the case with the fenestration above the renal artery caused the smallest decrease. A major limitation of our research is the lack of patient-specific geometries. Although we have previously validated our idealized model by comparing renal artery flow rates in our model with data reported in literature by Figueroa et al. [11], a more precise hemodynamic result would be obtained by studying models with patient-specific geometries applied with the same boundary conditions. 
TABLE I. MEAN Flow Rate OF Different TaKeOFF ANGLES AND Alignement CASES

\begin{tabular}{|c|c|c|c|c|c|}
\hline \multirow{3}{*}{ Takeoff Angle $\left(^{\circ}\right)$} & \multicolumn{5}{|c|}{ Mean Flow Rate (L/min) } \\
\hline & \multirow{2}{*}{ Aligned } & \multicolumn{2}{|c|}{ Vertical Misalignments } & \multicolumn{2}{|c|}{ Horizontal Misalignments } \\
\hline & & $2.8 \mathrm{~mm}$ Above & $2.8 \mathrm{~mm}$ Below & $17.5^{\circ}$ Clockwise & $17.5^{\circ}$ Counterclockwise \\
\hline 87.25 & 0.0223 & 0.0199 & 0.0207 & 0.0204 & 0.0204 \\
\hline
\end{tabular}

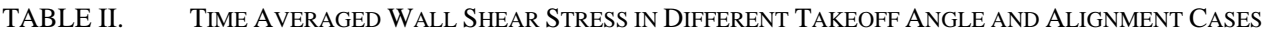

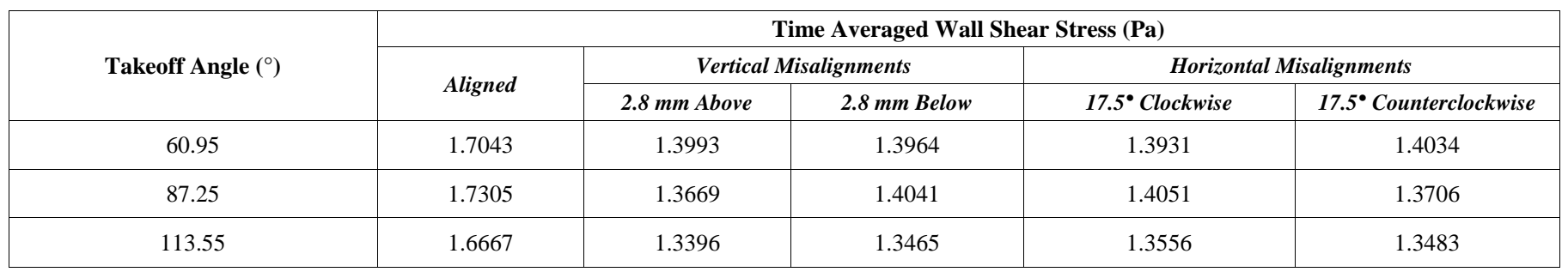

The study has built connection between fenestration misalignments and changes in hemodynamics, indicating that FEVAR with misalignment occurring could cause further renal dysfunction and possible post-operative impairment [4]. At the clinical level, it is suggested that closer attention should be put on possible fenestration misalignments when determining therapeutic strategies, and at the same time, more attention should be placed on studies to reduce misalignments.

\section{CONCLUSIONS}

Our study clearly showed that fenestration misalignments caused decreases of hemodynamic indices including renal artery flow rate and renal TAWSS. In the perspective of renal artery flow rate, among misalignments with same reduction of effective area, the case with the fenestration $2.8 \mathrm{~mm}$ below the renal artery caused the highest decrease in renal flow rate, while the case with the fenestration $2.8 \mathrm{~mm}$ above caused the lowest decrease of the index. The TOA also affects indices of renal artery flow rate. Cases with TOAs of $113.55^{\circ}$ had the lowest renal flow rate while cases with TOAs of $60.95^{\circ}$ had the highest. TOA has larger effects than misalignments on renal artery flow rate.

\section{REFERENCES}

[1] A. N. Assar and C. K. Zarins, "Ruptured abdominal aortic aneurysm: a surgical emergency with many clinical presentations," Postgrad. Med. J., vol. 85, pp. 268-273, 2009.

[2] M. G. A. Norwood, G. M. Lloyd, M. J. Bown, G. Fishwick, N. J. London, and R. D. Sayers, "Endovascular abdominal aortic aneurysm repair,” Postgrad. Med. J., vol. 83, pp. 21-27, 2007.

[3] A. N. Saratzis, M. F. Bath, S. C. Harrison, R. D. Sayers, and M. J. Bown, "Impact of fenestrated endovascular abdominal aortic aneurysm repair on renal function," Nephrol. Dial. Transplant., vol. 30, pp. 889-896, 2015.

[4] J. Ou, Y. C. Chan, C. Y. T. Chan, and S. W. Cheng, "Geometric alteration of renal arteries after fenestrated grafting and the impact on renal function,” Ann. Vasc. Surg., vol. 41, pp. 89-95, 2017.

[5] L. R. de Souza and G. S. Oderich, "Renal function deterioration in complex aortic repair," in Endovascular Aortic Repair: Current Techniques with Fenestrated, Branched and Parallel Stent-grafts, G. S. Oderich, Ed. Springer, 2017, pp. 726-728.

[6] V. Peiffer, S. J. Sherwin, and P. D. Weinberg, "Does low and oscillatory wall shear stress correlate spatially with early atherosclerosis? A systematic review," Cardiovasc. Res., vol. 99, pp. 242-250, 2013.

[7] H. Kandail, M. Hamady, and X. Y. Xu, "Comparison of blood flow in branched and fenestrated stent-grafts for endovascular repair of abdominal aortic aneurysms," J. Endovasc. Ther., vol. 22, pp. 578-590, 2015.

[8] D. Bluestein, L. Niu, R. T. Schoephoerster, and M. K. Dewanjee, "Steady flow in an aneurysm model: correlation between fluid dynamics and blood platelet deposition,” J. Biomech. Eng., vol. 118, p. 280, 1996.

[9] V. L. Rayz, L. Boussel, M. T. Lawton, G. Acevedo-Bolton, L. Ge, W. L. Young, R. T. Higashida, and D. Saloner, "Numerical modeling of the flow in intracranial aneurysms: prediction of regions prone to thrombus formation,” Ann. Biomed. Eng., vol. 36, pp. 1793-1804, 2008.

[10] M. Jackson, N. B. Wood, S. Zhao, A. Augst, J. H. Wolfe, W. M. W. Gedroyc, A. D. Hughes, S. A. M. Thom, and X. Y. Xu, "Low wall shear stress predicts subsequent development of wall hypertrophy in lower limb bypass grafts," Artery Res., vol. 3, pp. 32-38, 2009.

[11] C. A. Figueroa, C. A. Taylor, V. Yeh, A. J. Chiou, and C. K. Zarins, "Effect of curvature on displacement forces acting on aortic endografts: a 
3-dimensional computational analysis," J. Endovasc. Ther., vol. 16, pp. 284-294, 2009.

[12] J. R. Womersley, "Method for the calculation of velocity, rate of flow and viscous drag in arteries when the pressure gradient is known," J. Physiol. (Lond.), vol. 127, pp. 553-563, 1955.

[13] D. Chemla, E. M. Lau, Y. Papelier, P. Attal, and P. Hervé, "Pulmonary vascular resistance and compliance relationship in pulmonary hypertension,” Eur. Respir. J., vol. 46, pp. 1178-1189, 2015.

[14] E. Georgakarakos, A. Xenakis, G. S. Georgiadis, C. Argyriou, G. A. Antoniou, N. Schoretsanitis, and M. K. Lazarides, "The hemodynamic impact of misalignment of fenestrated endografts: A computational study," Eur. J. Vasc. Endovasc. Surg., vol. 47, pp. 151-159, 2014.

[15] H. Kandail, M. Hamady and X. Y. Xu, "Effect of a flared renal stent on the performance of fenestrated stent-grafts at rest and exercise conditions," J. Endovasc. Ther., vol. 23, pp. 809-820, 2016. 